\title{
Recyclable fluorous cinchona alkaloid ester as a chiral promoter for asymmetric fluorination \\ of $\beta$-ketoesters
}

\author{
Wen-Bin Yi ${ }^{* 1}$, Xin Huang ${ }^{2}$, Zijuan Zhang ${ }^{2}$, Dian-Rong Zhu ${ }^{2}$, Chun Cai ${ }^{1}$ \\ and Wei Zhang ${ }^{* 2}$
}

\section{Full Research Paper}

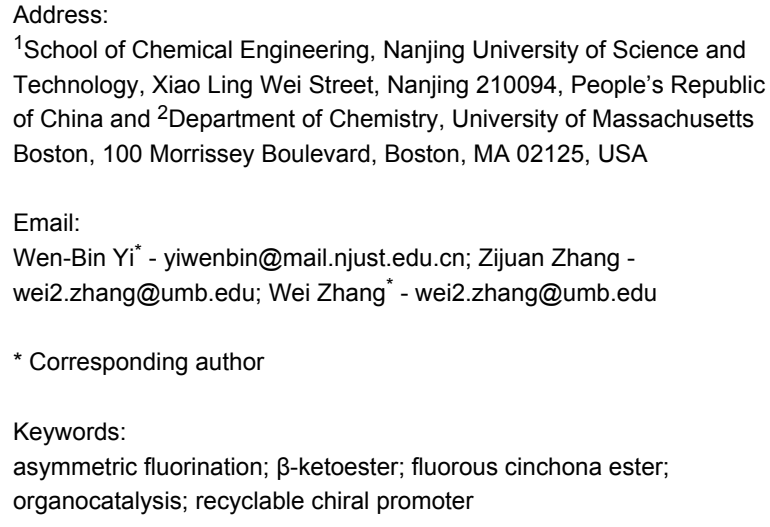

${ }^{1}$ School of Chemical Engineering, Nanjing University of Science and Technology, Xiao Ling Wei Street, Nanjing 210094, People's Republic of China and ${ }^{2}$ Department of Chemistry, University of Massachusetts Boston, 100 Morrissey Boulevard, Boston, MA 02125, USA

\section{Email:}

Wen-Bin Yi ${ }^{*}$ - yiwenbin@mail.njust.edu.cn; Zijuan Zhang wei2.zhang@umb.edu; Wei Zhang ${ }^{*}$ - wei2.zhang@umb.edu

* Corresponding author

Keywords:

asymmetric fluorination; $\beta$-ketoester; fluorous cinchona ester; organocatalysis; recyclable chiral promoter

\begin{abstract}
A fluorous cinchona alkaloid ester has been developed as a chiral promoter for the asymmetric fluorination of $\beta$-ketoesters. It has comparable reactivity and selectivity to the nonfluorous versions of cinchona alkaloids and can be easily recovered from the reaction mixture by simple fluorous solid-phase extraction (F-SPE) and used for the next round of reaction without further purification.
\end{abstract}

\section{Introduction}

Fluorinated organic compounds have unique properties because fluorine forms a strong carbon-fluorine bond with a small covalent radius and high electronegativity. Other than fluorinated polymers in materials science, organofluorine compounds have gained increasing popularity in medical chemistry and agricultural chemistry. Introducing one or a few fluorine atoms to biologically interesting molecules can significantly change the physical, chemical and biological properties $[1,2]$. The significant amount of publications on fluorinated small molecules, amino acids, carbohydrates, steroids and nucleosides indicates

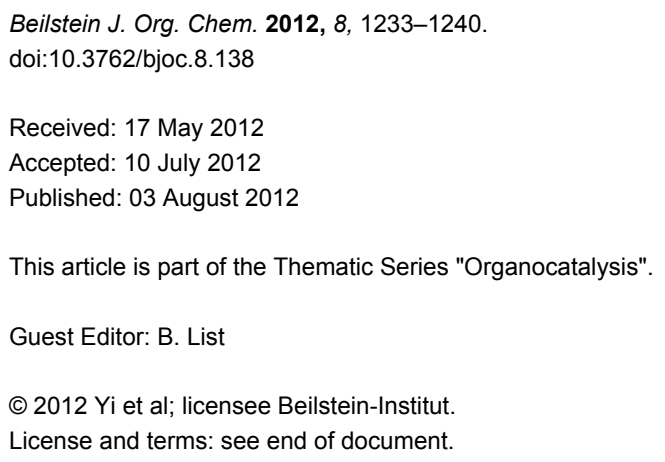

that organofluorine chemistry plays an important role in the life sciences $[3,4]$.

A fluorine atom has been introduced to the $\alpha$-position of some biologically interesting $\beta$-ketoesters, such as erythromycin and sesquiterpenic drimane (Figure 1) [5,6]. The achiral fluorination of $\beta$-ketoesters can be achieved by electrophilic reaction with Selectfluor (F-TEDA-BF 4 , 1-chloromethyl-4-fluoro-1,4diazoniabicyclo[2.2.2] octane bis(tetrafluoroborate)), as developed by Bank [7-9]. The Cahard [10-12] and Shibata [13,14] 
groups combined cinchona alkaloids and Selectfluor for asymmetric fluorination of substrates such as imido-protected phenylglycines (up to $94 \%$ ee), indanones and tetralones (up to $91 \%$ ee), ethyl $\alpha$-cyanotolyl acetates (up to $87 \%$ ee), and cyclic $\beta$-ketoesters (up to $80 \%$ ee) [15]. A catalytic approach for the cinchona alkaloids and Selectfluor combinations has also been developed [16]. The Togni group employed chiral titanium Lewis acid $\mathrm{TiCl}_{2}$ (TADDOLate) for the asymmetric fluorination of $\beta$-ketoesters (up to 96\% ee) [17-20]. Most Selectfluorpromoted asymmetric fluorinations require a stoichiometric amount of chiral promoters to suppress the competitively direct achiral fluorination. Different supported cinchona alkaloids have been developed as recyclable chiral promoters or organocatalysts. Among them, the Cahard group developed soluble polymer- and ionic-liquid-supported cinchona alkaloids for electrophilic fluorination [21,22]. The Fache and Soós groups developed fluorous tag-attached cinchona alkaloids for catalytic Diels-Alder reactions $[23,24]$. Introduced in this paper is a new fluorous cinchona alkaloid ester for flourination of $\beta$-ketoesters. It is part of our recent effort on the development of recyclable fluorous reagents and organocatalysts for asymmetric synthesis [25-27].

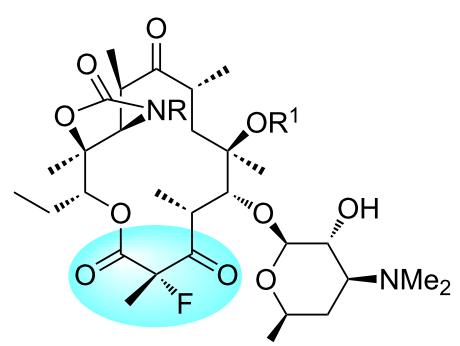

erythromycin derivatives (antibacterial)

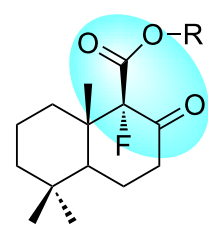

fluorinated drimanes (antimalarial)
Figure 1: Biologically interesting a-fluorinated $\beta$-ketoesters.

\section{Results and Discussion}

Cinchona alkaloids and their derivatives have been wellexplored in asymmetric synthesis [28]. We envisioned that the introduction of a fluorous tag could facilitate the recycling of cinchona alkaloids. The synthesis of fluorous quinine ester $\mathbf{C - 1}$ was accomplished by the reaction of quinine with a fluorous acid chloride (Scheme 1). This compound was easily purified by fluorous-solid phase extraction (F-SPE) with a cartridge charged with fluorous silica gels [29,30]. It is stable in air and soluble in solvents such as $\mathrm{CH}_{2} \mathrm{Cl}_{2}, \mathrm{CH}_{3} \mathrm{OH}$, and $\mathrm{CH}_{3} \mathrm{CN}$.

With the fluorous quinine ester $\mathbf{C}-\mathbf{1}$ in hand, we explored the fluorination reaction using ethyl 2-methyl-3-oxo-3-phenylpropanoate (1a) as a model compound. Nonfluorous quinine esters, such as $\mathbf{C - 2}$ and $\mathbf{C - 3}$, cinchona alkaloids $\mathbf{C - 4}$ and $\mathbf{C - 5}$, and fluorous pyrrolidine ester $\mathbf{C - 6}$, were also evaluated (Figure 2). The results of the fluorination of $\beta$-ketoester 1a with Selectfluor and different promoters are listed in Table 1. It was found that using $\mathrm{MeCN}$ as a solvent with 1 equiv of $\mathbf{C - 1}$ gave fluorinated product $\mathbf{2 a}$ in $49 \%$ yield and $65 \%$ ee (Table 1 , entry 1). Compared to other promoters (Table 1, entries 2-5), C-1 gave fluorinated products in a slightly low yield but better enantioselectivity. This may be attributed to the stereo and the electronic effect of the fluorous tag. Fluorous pyrrolidine C-6 (Table 1, entry 6) gave the lowest product yield and ee among all six promoters. Reducing the amount of $\mathbf{C}-\mathbf{1}$ from 1 equiv to 0.5 and 0.2 equiv significantly reduced the ee of the product (Table 1, entries 7 and 8). A control reaction without $\mathbf{C - 1}$ gave 2a in $35 \%$ yield as a racemic product (Table 1, entry 9). The results suggest that a stoichiometric amount of $\mathbf{C - 1}$ is required to minimize the formation of achiral fluorination product by direct fluorination. Solvent screening indicated that using 1:1 $\mathrm{CH}_{3} \mathrm{CN} / \mathrm{CH}_{2} \mathrm{Cl}_{2}$ gave product $2 \mathrm{a}$ in $51 \%$ yield and $70 \%$ ee (Table 1, entry 15), which is better than using $\mathrm{CH}_{3} \mathrm{CN}$ alone. Other single or binary solvent systems containing toluene, THF, $\mathrm{H}_{2} \mathrm{O}$, and $\mathrm{CF}_{3} \mathrm{C}_{6} \mathrm{H}_{5}$ did not afford better results (Table 1, entries $10-14)$. It was also found that lowering of the reaction temperature from 25 to 10 or $0{ }^{\circ} \mathrm{C}$ did not necessarily improve the enantioselectivity of the fluorination (Table 1, entries 16 and 17).

Recycling of promoter $\mathbf{C - 1}$ is an important part of this project. In our previous work we have demonstrated that fluorous organocatalysts and reagents can be readily recovered by F-SPE<smiles>C=CC1CN2CCC1C2[C@H](O)c1ccnc2ccc(OC)cc12</smiles>

$+$<smiles>O=C(Cl)c1ccc(CCc2ccc(F)cc2)cc1</smiles>

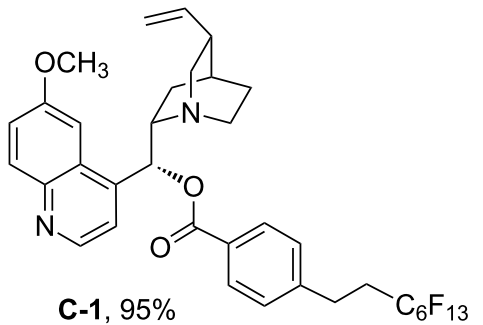


<smiles>C=CC1CN2CCC1C([C@H](OC(=O)c1ccc(CCC(F)F)cc1)c1ccnc3ccc(OC)cc13)C2</smiles>

C-1<smiles>C=CC1CN2CCC1C2[C@H](O)c1ccnc2ccc(OC)cc12</smiles>

C-4<smiles>C=CC1CC2CCN1CC2[C@H](OC(=O)c1ccccc1)c1ccnc2ccc(OC)cc12</smiles>

C-2<smiles>CCC1CN2CCC1C2[C@H](O)c1ccnc2ccc(OC)cc12</smiles>

C-5<smiles>C=CC1CC2CCN1C2[C@H](OC(C)=O)c1ccnc2ccc(OC)cc12</smiles>

C-3

C-6

Figure 2: Promoters for asymmetric fluorination.

Table 1: Asymmetric fluorination of $1 a^{a}$

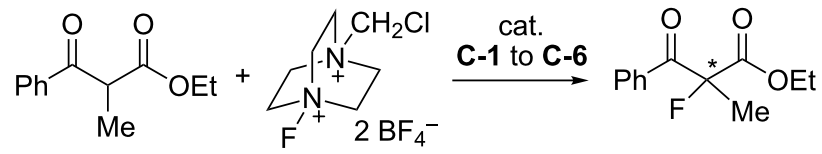

$1 \mathrm{a}$

Selectfluor

$2 a$

\begin{tabular}{|c|c|c|c|c|c|}
\hline Entry & Cat. (equiv) & Solvent & $t(\mathrm{~h})$ & Yield (\%) & ee $(\%)$ \\
\hline 1 & C-1 (1.0) & $\mathrm{MeCN}$ & 72 & 49 & 65 \\
\hline 2 & C-2 (1.0) & $\mathrm{MeCN}$ & 72 & 52 & 56 \\
\hline 3 & C-3 (1.0) & $\mathrm{MeCN}$ & 72 & 54 & 51 \\
\hline 4 & C-4 (1.0) & $\mathrm{MeCN}$ & 72 & 62 & 46 \\
\hline 5 & C-5 (1.0) & $\mathrm{MeCN}$ & 72 & 65 & 48 \\
\hline 6 & C-6 (1.0) & $\mathrm{MeCN}$ & 96 & 41 & 18 \\
\hline 7 & C-1 (0.5) & $\mathrm{MeCN}$ & 60 & 51 & 26 \\
\hline 8 & C-1 (0.2) & $\mathrm{MeCN}$ & 60 & 41 & $<5$ \\
\hline 9 & - & $\mathrm{MeCN}$ & 96 & 35 & 0 \\
\hline 10 & C-1 (1.0) & Toluene & 72 & 16 & 23 \\
\hline 11 & C-1 (1.0) & THF & 72 & 32 & 41 \\
\hline 12 & C-1 (1.0) & $\mathrm{H}_{2} \mathrm{O}$ & 96 & - & - \\
\hline 13 & C-1 (1.0) & $\mathrm{MeCN} / \mathrm{THF}$ & 60 & 38 & 45 \\
\hline 14 & C-1 (1.0) & $\mathrm{MeCN} / \mathrm{CF}_{3} \mathrm{C}_{6} \mathrm{H}_{5}$ & 60 & 43 & 59 \\
\hline 15 & C-1 (1.0) & $\mathrm{MeCN} / \mathrm{CH}_{2} \mathrm{Cl}_{2}$ & 60 & 51 & 70 \\
\hline $16^{b}$ & C-1 (1.0) & $\mathrm{MeCN} / \mathrm{CH}_{2} \mathrm{Cl}_{2}$ & 72 & 46 & 69 \\
\hline $17^{c}$ & C-1 (1.0) & $\mathrm{MeCN} / \mathrm{CH}_{2} \mathrm{Cl}_{2}$ & 72 & 39 & 71 \\
\hline
\end{tabular}

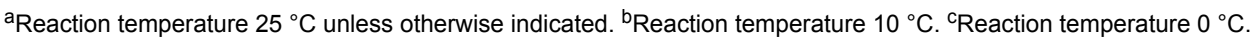

$[19,20]$. In the current work, upon completion of the fluorination reaction, a base such as aqueous $\mathrm{NaOH}$ or $\mathrm{KOH}$ was added to the reaction mixture to convert the cinchona alkaloid/ Selectfluor complex to free cinchona alkaloid. The organic phase was loaded onto a fluorous silica gel cartridge for F-SPE. Promoter C-1 was recovered in high yield (94\%) and excellent purity (98\%). It was used for five rounds without significant change of product yield and ee (Scheme 2). 


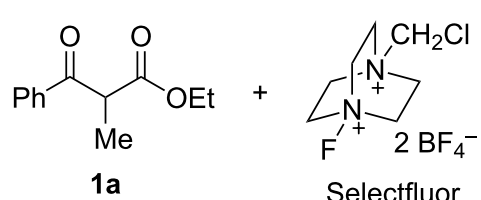

1a
$\stackrel{\mathrm{MeCN} / \mathrm{CH}_{2} \mathrm{Cl}_{2}}{25^{\circ} \mathrm{C}}$
$\underset{\mathrm{C}-1}{\mathrm{~F}-\mathrm{SPE} \text { recovery }}$<smiles>CCOC(=O)C(F)(F)C(=O)c1ccccc1</smiles>

2a

$1^{\text {st. }} 51 \%$ yield, $70 \%$ ee

$2^{\text {nd }}: 48 \%$ yield, $69 \%$ ee

$3^{\text {rd: }} 47 \%$ yield, $69 \%$ ee

$4^{\text {th: }} 47 \%$ yield, $68 \%$ ee

$5^{\text {th }}: 46 \%$ yield, $67 \%$ ee

Scheme 2: Preparation of 2a by using recycled quinine ester $\mathbf{C}-\mathbf{1}$.

The scope of fluorous quinine ester C-1-mediated fluorination was evaluated by carrying out the reactions with a number of $\alpha$-substituted ethyl benzoylacetates $\mathbf{1 a - e}$ and $\mathbf{1 g - i}$ as well as ethyl 2-cyclohexanonecarboxylate (1f). Results summarized in Figure 3 indicate that benzoylacetates bearing $\mathrm{R}^{2}$ such as $\mathrm{Me}$, $\mathrm{PhCH}_{2}, \mathrm{Cl}$, and $\mathrm{Br}$ gave fluorination products $\mathbf{2 a - d}$ in $43-71 \%$ yields and $60-70 \%$ ee. The nonsubstituted benzoylacetate 1e gave product $2 \mathrm{e}$ in good yield $69 \%$ but low ee $(31 \%)$. Ethyl 2-cyclohexanonecarboxylate (1f) afforded product $\mathbf{2 f}$ in $73 \%$ yield and $63 \%$ ee. Reactions of ethyl benzoylacetates with bigger substitution groups, such as phenylsulfonyl and maleimide derivatives, were also attempted and gave products $\mathbf{2 g}-\mathbf{i}$ in $74-83 \%$ yields and $78-81 \%$ ee.

\section{Conclusion}

A fluorous cinchona alkaloid-ester has been introduced as a promoter for Selectfluor-based asymmetric fluorination of $\beta$-ketoesters. The fluorous promoter has slightly lower reactivity but better enantioselectivity than the nonfluorous cinchona alkaloids. It can be easily recovered by simple fluorous solidphase extraction for reuse.

\section{Experimental}

\section{General}

Chemicals and solvents were purchased from commercial suppliers and used as received. ${ }^{1} \mathrm{H}$ and ${ }^{13} \mathrm{C}$ NMR spectra were recorded on a $300 \mathrm{MHz}$ Varian NMR spectrometer. Chemical<smiles>[R]C(=O)C([R])CC</smiles>

2a, $60 \mathrm{~h}, 51 \%$ yield, $70 \%$ ee<smiles>CCOC(=O)C(F)(Br)C(=O)c1ccccc1</smiles>

2d, 72 h, $43 \%$ yield, $66 \%$ ee<smiles>CCOC(=O)[C@@](F)(C(=O)c1ccccc1)c1ccc(C)cc1</smiles>

2g, 64 h, $74 \%$ yield, $78 \%$ ee<smiles>F[N+]12CCN(CC1)CC2[P+](F)(F)Br</smiles>

Selectfluor

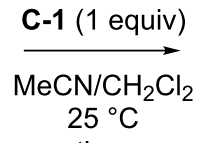

time<smiles>CCOC(=O)C(C)(F)C(=O)c1ccccc1</smiles>

2b, 72 h, $59 \%$ yield, $60 \%$ ee<smiles>CCOC(=O)C(F)C(=O)c1ccccc1</smiles>

2e, $48 \mathrm{~h}, 69 \%$ yield, $31 \%$ ee<smiles>CCOC(=O)[C@@](F)(C(=O)c1ccccc1)[C@H]1CC(=O)N(CC)C1=O</smiles>

2h, 72 h, $78 \%$ yield, $82 \%$ ee<smiles>[R]C(=O)C([R])(F)C(=O)OCC</smiles><smiles>CCOC(=O)C(F)(Cl)C(=O)c1ccccc1</smiles>

2c, 32 h, $71 \%$ yield, $66 \%$ ee<smiles>CCOC(=O)C1(F)CCCCC1=O</smiles>

2f, $72 \mathrm{~h}, 73 \%$ yield, $63 \%$ ee<smiles>CCOC(=O)[C@@](F)(C(=O)c1ccccc1)[C@H]1CC(=O)N(Cc2ccccc2)C1=O</smiles>

2i, 72 h, $83 \%$ yield, $81 \%$ ee

Figure 3: The asymmetric fluorination of various $\beta$-ketoesters. 
shifts were reported in parts per million (ppm), and the residual solvent peak was used as an internal reference, i.e., proton (chloroform $\delta$ 7.26), carbon (chloroform $\delta 77.0$ ). Multiplicity was indicated as follows: $s$ (singlet), $d$ (doublet), $t$ (triplet), $q$ (quartet), m (multiplet), dd (doublet of doublet), br s (broad singlet). Coupling constants were reported in hertz $(\mathrm{Hz})$. LC-MS were performed on an Agilent 2100 system. A C18 column $(5.0 \mu \mathrm{m}, 6.0 \times 50 \mathrm{~mm})$ was used for the separation. The mobile phases were methanol and water, both containing $0.05 \%$ trifluoroacetic acid. A linear gradient was used to increase from $25: 75 \mathrm{v} / \mathrm{v}$ methanol/water to $100 \%$ methanol over $7.0 \mathrm{~min}$ at a flow rate of $0.7 \mathrm{~mL} / \mathrm{min}$. UV detections were conducted at 210 , 254 and $365 \mathrm{~nm}$. Low-resolution mass spectra were recorded in APCI (atmospheric pressure chemical ionization). The highresolution mass spectra were obtained on a Finnigan/MAT 95XL-T spectrometer. Sorbent silica gel XHL TLC plates (130815) were used for the thin-layer chromatography (TLC). Flash chromatography separations were performed on YAMAZEN AI-580 flash column system with Agela silica gel columns (230-400 $\mu \mathrm{m}$ mesh). The enantiomeric excesses of products were determined by chiral phase HPLC analysis on an SHIMADZU LC-20AD system.

\section{Synthesis of fluorous quinine ester C-1}

Thionyl chloride $(1.19 \mathrm{~g}, 10 \mathrm{mmol})$ was added to a mixture of ( $1 H, 1 H, 2 H, 2 H$-perfluorooctyl)benzoic acid $(0.468 \mathrm{~g}, 1 \mathrm{mmol})$ and pyridine $(75 \mathrm{mg}, 1 \mathrm{mmol})$. After stirring of the mixture for $4 \mathrm{~h}$ at $50^{\circ} \mathrm{C}$, the reaction container was flushed with nitrogen gas to remove unreacted thionyl chloride. Quinine $(0.275 \mathrm{~g}$, $0.85 \mathrm{mmol})$ and $N, N$-diisopropylethylamine (129 mg, $1 \mathrm{mmol})$ in $\mathrm{CH}_{2} \mathrm{Cl}_{2}(3 \mathrm{~mL})$ was added, and the solution was stirred for $24 \mathrm{~h}$ under reflux. After the reaction had been quenched with $\mathrm{H}_{2} \mathrm{O}(2 \mathrm{~mL})$ for $1 \mathrm{~h}$, aqueous $\mathrm{K}_{2} \mathrm{CO}_{3}(2 \mathrm{M}, 10 \mathrm{~mL})$ was added, and the mixture was extracted with $\mathrm{CH}_{2} \mathrm{Cl}_{2}(3 \times 10 \mathrm{~mL})$. The $\mathrm{CH}_{2} \mathrm{Cl}_{2}$ layer was washed with aqueous $\mathrm{HCl}$ (ca. $2 \mathrm{M}, 10 \mathrm{~mL}$ ) and $\mathrm{H}_{2} \mathrm{O}(20 \mathrm{~mL})$. The combined extracts were dried over $\mathrm{K}_{2} \mathrm{CO}_{3}$ and evaporated. The slightly yellow residue was purified by a fluorous silica gel cartridge $(5 \mathrm{~g})$. It was first eluted with $80: 20 \mathrm{MeOH} / \mathrm{H}_{2} \mathrm{O}(20 \mathrm{~mL})$ and then with $100 \% \mathrm{MeOH}$. The $\mathrm{MeOH}$ fraction was concentrated to give $\mathbf{C - 1}$ as a yellowish solid (0.625 g, 95\%). Mp $175-177{ }^{\circ} \mathrm{C} ;{ }^{1} \mathrm{H}$ NMR $\left(\mathrm{CDCl}_{3}, 300 \mathrm{MHz}\right) \delta 1.51-2.05(\mathrm{~m}, 6 \mathrm{H}), 2.30-2.42(\mathrm{~m}, 3 \mathrm{H})$, 2.65-2.70 (m, 2H), 2.97-3.18 (m, 4H), 3.50 (q, $J=6.9 \mathrm{~Hz}, 1 \mathrm{H})$, $3.98(\mathrm{~s}, 3 \mathrm{H}), 5.02(\mathrm{~m}, 2 \mathrm{H}), 5.83(\mathrm{~m}, 1 \mathrm{H}), 6.72(\mathrm{~d}, J=6.9 \mathrm{~Hz}$, $1 \mathrm{H}), 7.32-7.51(\mathrm{~m}, 5 \mathrm{H}), 8.01-8.07(\mathrm{~m}, 3 \mathrm{H}), 8.72-8.73(\mathrm{~d}, 1 \mathrm{H})$; ${ }^{13} \mathrm{C} \mathrm{NMR}\left(\mathrm{CDCl}_{3}, 75 \mathrm{MHz}\right) \delta 24.2,26.5,27.6,27.9,32.4,39.7$, 42.6, 55.6, 56.7, 59.4, 74.5, 101.3, 114.6, 117.3, 118.6, 121.9, $126.9,128.3,128.63,130.2,131.9,141.7,143.6,144.8,145.0$, 147.5, 156.0, 165.3; APCIMS $m / z$ : $775.1\left(\mathrm{M}^{+}+1\right)$; HRMS-ESI $(m / z):[\mathrm{M}+\mathrm{H}]^{+}$calcd. for $\mathrm{C}_{35} \mathrm{H}_{32} \mathrm{~F}_{13} \mathrm{~N}_{2} \mathrm{O}_{3}, 775.2205$; found, 775.2214 .

\section{Synthesis of quinine benzoate catalyst $\mathbf{C}$-2}

Benzoyl chloride ( $28 \mathrm{mg}, 0.2 \mathrm{mmol}$ ) was added to a mixture of quinine (65 mg, $0.2 \mathrm{mmol})$ in $\mathrm{CH}_{2} \mathrm{Cl}_{2}(0.5 \mathrm{~mL})$. After stirring at rt for $4 \mathrm{~h}$, aqueous $\mathrm{K}_{2} \mathrm{CO}_{3}(2 \mathrm{M}, 1 \mathrm{~mL})$ was added. The reaction mixture was extracted with $\mathrm{CH}_{2} \mathrm{Cl}_{2}(2 \times 3 \mathrm{~mL})$. The $\mathrm{CH}_{2} \mathrm{Cl}_{2}$ layer was washed with aqueous $\mathrm{HCl}(2 \mathrm{M}, 2 \mathrm{~mL})$ and $\mathrm{H}_{2} \mathrm{O}(3 \mathrm{~mL})$. The combined organic extracts were dried $\left(\mathrm{K}_{2} \mathrm{CO}_{3}\right)$ and evaporated. The white residue was purified by flash column chromatography $\left(18: 1 \mathrm{CH}_{2} \mathrm{Cl}_{2} / \mathrm{MeOH}\right)$ to give quinine benzoate $\mathbf{C - 2}(77 \mathrm{mg}, 90 \%)$ as a colorless solid. ${ }^{1} \mathrm{H} \mathrm{NMR}\left(\mathrm{CDCl}_{3}, 300 \mathrm{MHz}\right) \delta 1.69-2.00(\mathrm{~m}, 5 \mathrm{H}), 2.42(\mathrm{~m}$, $1 \mathrm{H}), 2.82(\mathrm{~m}, 2 \mathrm{H}), 3.19-3.40(\mathrm{~m}, 2 \mathrm{H}), 3.49-3.56(\mathrm{q}, J=7.2 \mathrm{~Hz}$, $1 \mathrm{H}), 4.00(\mathrm{~s}, 3 \mathrm{H}), 5.04(\mathrm{~m}, 2 \mathrm{H}), 5.82(\mathrm{~m}, 1 \mathrm{H}), 6.97(\mathrm{~d}, J=7.2$, $1 \mathrm{H}), 7.40-7.65(\mathrm{~m}, 6 \mathrm{H}), 8.01-8.13(\mathrm{~m}, 3 \mathrm{H}), 8.73(\mathrm{~d}, 1 \mathrm{H})$; ${ }^{13} \mathrm{C} \mathrm{NMR}\left(\mathrm{CDCl}_{3}, 75 \mathrm{MHz}\right) \delta 23.1,27.2,27.5,39.0,42.5,56.0$, 56.1, 59.0, 73.5, 101.2, 115.2, 117.2, 122.3, 126.6, 127.9, 128.7, 129.6, 129.6, 131.6, 131.8, 133.6, 140.6, 144.7, 147.2, 158.3, 165.1, 200.2; APCIMS m/z: $429.2\left(\mathrm{M}^{+}+1\right)$.

\section{Synthesis of quinine acetate $\mathbf{C}-\mathbf{3}$}

Acetic anhydride $(30 \mathrm{mg}, 0.3 \mathrm{mmol})$ was added to a mixture of quinine ( $65 \mathrm{mg}, 0.2 \mathrm{mmol}$ ) in $\mathrm{CH}_{2} \mathrm{Cl}_{2}(0.5 \mathrm{~mL})$. After stirring at rt for $8 \mathrm{~h}$, aqueous $\mathrm{K}_{2} \mathrm{CO}_{3}(2 \mathrm{M}, 1 \mathrm{~mL})$ was added, and the mixture was extracted with $\mathrm{CH}_{2} \mathrm{Cl}_{2}(2 \times 3 \mathrm{~mL})$. The $\mathrm{CH}_{2} \mathrm{Cl}_{2}$ layer was washed with aqueous $\mathrm{HCl}(2 \mathrm{M}, 2 \mathrm{~mL})$ and $\mathrm{H}_{2} \mathrm{O}(3 \mathrm{~mL})$. The combined organic extracts were dried $\left(\mathrm{K}_{2} \mathrm{CO}_{3}\right)$ and evaporated. The white residue was purified by flash column chromatography $\left(18: 1 \mathrm{CH}_{2} \mathrm{Cl}_{2} / \mathrm{MeOH}\right)$ to give quinine acetate $\mathbf{C - 3}$ (67 mg, 92\%) as a colorless oil. ${ }^{1} \mathrm{H} \mathrm{NMR}\left(\mathrm{CDCl}_{3}, 300 \mathrm{MHz}\right) \delta$ 1.26-1.89 (m, 5H), $2.42(\mathrm{~m}, 1 \mathrm{H}), 2.12(\mathrm{~s}, 3 \mathrm{H}), 2.23-2.36(\mathrm{~m}$, $2 \mathrm{H}), 2.37-2.70(\mathrm{~m}, 2 \mathrm{H}), 3.00-3.16(\mathrm{~m}, 2 \mathrm{H}), 3.34-3.42(\mathrm{q}, J=$ $7.2 \mathrm{~Hz}, 1 \mathrm{H}), 3.96(\mathrm{~s}, 3 \mathrm{H}), 5.03(\mathrm{~m}, 2 \mathrm{H}), 5.86(\mathrm{~m}, 1 \mathrm{H}), 6.50(\mathrm{~d}, J$ $=7.2 \mathrm{~Hz}, 1 \mathrm{H}), 7.35-7.44(\mathrm{~m}, 3 \mathrm{H}), 8.02(\mathrm{~d}, J=9.0 \mathrm{~Hz}, 3 \mathrm{H}), 8.74$ $(\mathrm{d}, 1 \mathrm{H}) ;{ }^{13} \mathrm{C} \mathrm{NMR}\left(\mathrm{CDCl}_{3}, 75 \mathrm{MHz}\right) \delta 21.1,24.3,27.5,27.7$, 39.6, 42.4, 55.6, 56.5, 59.0, 73.7, 101.4, 114.5, 118.9, 121.8, 127.0, 131.8, 141.7, 143.5, 144.8, 147.4, 149.6, 157.9, 170.0, 199.5, 200.2; ACPIMS $m / z$ : $367.2\left(\mathrm{M}^{+}+1\right)$.

\section{Synthesis of fluorous pyrrolidine ester C-6}

$N, N^{\prime}$-Dicyclohexylcarbodiimide (DCC) $(0.206 \mathrm{~g}, 1 \mathrm{mmol})$ was added to a mixture of $(1 H, 1 H, 2 H, 2 H$-perfluorooctyl)benzoic acid (0.468 g, $1 \mathrm{mmol}), N$-Boc-L-prolinol $(0.221 \mathrm{~g}, 1.1 \mathrm{mmol})$, 4-dimethylaminopyridine (DMAP) $(0.122 \mathrm{~g}, 1 \mathrm{mmol})$ in THF. After being stirred for $24 \mathrm{~h}$ at $\mathrm{rt}$, the mixture was directly loaded onto a fluorous silica-gel cartridge ( $5 \mathrm{~g}$; eluted by $100 \%$ methanol) to give the $\mathrm{N}$-Boc-L-prolinyl $(1 \mathrm{H}, 1 \mathrm{H}, 2 \mathrm{H}, 2 \mathrm{H}$-perfluorooctyl)benzoate $(0.618 \mathrm{~g}, 95 \%)$. The $N$-Boc ester was then added to a mixture of TFA in $\mathrm{CH}_{2} \mathrm{Cl}_{2}$. After being stirred for $12 \mathrm{~h}$ at $0{ }^{\circ} \mathrm{C}$, the reaction mixture was loaded onto a fluorous silica-gel cartridge $(5 \mathrm{~g})$ again to give the title compound L-prolinyl $(1 H, 1 H, 2 H, 2 H$-perfluorooctyl)benzoate $(0.496 \mathrm{~g}$, 
90\%). ${ }^{1} \mathrm{H} \mathrm{NMR}\left(\mathrm{CDCl}_{3}, 300 \mathrm{MHz}\right) \delta 1.62-1.89(\mathrm{~m}, 3 \mathrm{H})$, 2.16-2.21 (m, 1H), 2.29-2.47 (m, 2H), 2.92-2.98 (m, 2H), $3.48-3.54(\mathrm{~m}, 2 \mathrm{H}), 3.71-3.84(\mathrm{~m}, 2 \mathrm{H}), 4.38-4.42(\mathrm{~m}, 1 \mathrm{H})$, 4.95-4.97 (m, 2H), 7.27-7.31 (d, 2H), 7.48-7.50 (d, 2H); ${ }^{13} \mathrm{C} \mathrm{NMR}\left(\mathrm{CDCl}_{3}, 75 \mathrm{MHz}\right) \delta 25.0,26.2,26.3,26.3,28.5,32.2$, 32.6, 51.1, 61.6, 67.2, 127.3, 127.6, 128.3, 128.3, 128.4, 130.1, 135.1, 141.4, 171.9; APCIMS m/z: $552.1\left(\mathrm{M}^{+}+1\right)$.

\section{General procedure for fluorination reaction}

A mixture of Selectfluor $(0.057 \mathrm{~g}, 0.16 \mathrm{mmol})$ and fluorous quinine ester $\mathbf{C}-1$ ( $0.124 \mathrm{~g}, 0.16 \mathrm{mmol})$ in $\mathrm{CH}_{3} \mathrm{CN}$ and $\mathrm{CH}_{2} \mathrm{Cl}_{2}$ was stirred at $\mathrm{rt}$ for $1 \mathrm{~h}$. Ethyl 2-methyl-3-oxo-3-phenylpropanoate (1a) (0.033 g, $0.16 \mathrm{mmol})$ was added. After stirring of the mixture at $\mathrm{rt}$ for $32 \mathrm{~h}$, the reaction was quenched with $\mathrm{H}_{2} \mathrm{O}$. After F-SPE, the mixture was extracted with EtOAc. The organic layer was washed with aqueous $\mathrm{HCl}(2 \mathrm{M}, 5 \mathrm{~mL})$ and $\mathrm{H}_{2} \mathrm{O}(5 \mathrm{~mL})$, and then dried over $\mathrm{Na}_{2} \mathrm{SO}_{4}$. After evaporation of the solvent, the residue was purified by flash column chromatography (8:1 hexane/EtOAc) to give $(S)$-ethyl 2-methyl-2fluoro-3-oxo-3-phenylpropanoate (2a) as a colorless oil.

\section{(S)-Ethyl 2-methyl-2-fluoro-3-oxo-3-phenyl- propanoate $(\mathbf{2 a})$}

$51 \%$ yield, $70 \%$ ee. The enantiomeric excess was determined by HPLC on (R,R)-WHELK-O1 with hexane/iPrOH (92:8) as the eluent. Flow rate: $0.6 \mathrm{~mL} / \mathrm{min}, \lambda=254 \mathrm{~nm} ; t_{\text {minor }}=20.132$ min, $t_{\text {major }}=17.924 \mathrm{~min} ;{ }^{1} \mathrm{H} \mathrm{NMR}\left(\mathrm{CDCl}_{3}, 300 \mathrm{MHz}\right) \delta 1.00$ (t, $J=7.2 \mathrm{~Hz}, 3 \mathrm{H}), 1.93(\mathrm{~s}, 1 \mathrm{H}), 4.11$ (q, $J=7.2 \mathrm{~Hz}, 2 \mathrm{H})$, 7.33-7.38 (m, 2H), $7.46(\mathrm{~m}, 1 \mathrm{H}), 7.90-7.92$ (m, 2H); APCIMS $m / z: 225.2\left(\mathrm{M}^{+}+1\right)$.

\section{(S)-Ethyl 2-benzyl-2-fluoro-3-oxo-3-phenyl- propanoate $(\mathbf{2 b})$}

$59 \%$ yield, $60 \%$ ee. The enantiomeric excess was determined by HPLC on Regis Chiral 5 Micron with hexane/iPrOH (90:10) as the eluent. Flow rate: $0.8 \mathrm{~mL} / \mathrm{min}, \lambda=254 \mathrm{~nm} ; t_{\text {minor }}=$ $8.732 \mathrm{~min}, t_{\text {major }}=10.352 \mathrm{~min} ;{ }^{1} \mathrm{H} \mathrm{NMR}\left(\mathrm{CDCl}_{3}, 300 \mathrm{MHz}\right) \delta$ $0.92(\mathrm{t}, J=7.2 \mathrm{~Hz}, 3 \mathrm{H}), 3.48$ (d, $J=14.1 \mathrm{~Hz}, 1 \mathrm{H}), 3.67$ (d, $J=$ $14.1 \mathrm{~Hz}, 1 \mathrm{H}), 4.01$ (q, $J=7.2 \mathrm{~Hz}, 2 \mathrm{H}), 7.14-7.23(\mathrm{~m}, 5 \mathrm{H}), 7.26$ $(\mathrm{m}, 2 \mathrm{H}), 7.36(\mathrm{~m}, 1 \mathrm{H}), 7.91(\mathrm{~d}, 2 \mathrm{H})$; APCIMS $m / z: 301.1$ $\left(\mathrm{M}^{+}+1\right)$.

\section{$(R)$-Ethyl 2-chloro-2-fluoro-3-oxo-3-phenyl- propanoate (2c)}

$71 \%$ yield, $66 \%$ ee. The enantiomeric excess was determined by HPLC on Regis Chiral 5 Micron with hexane/iPrOH (90:10) as the eluent. Flow rate: $0.8 \mathrm{~mL} / \mathrm{min}, \lambda=254 \mathrm{~nm} ; t_{\text {minor }}=$ $12.220 \mathrm{~min}, t_{\text {major }}=14.492 \mathrm{~min} ;{ }^{1} \mathrm{H} \mathrm{NMR}\left(\mathrm{CDCl}_{3}, 300 \mathrm{MHz}\right) \delta$ $1.18(\mathrm{t}, J=7.2 \mathrm{~Hz}, 3 \mathrm{H}), 4.32$ (q, $J=7.2 \mathrm{~Hz}, 2 \mathrm{H}), 7.47-7.49$ (m, $2 \mathrm{H}), 7.60(\mathrm{~m}, 1 \mathrm{H}), 8.02-8.05(\mathrm{~m}, 2 \mathrm{H})$; APCIMS $m / z: 245.0$ $\left(\mathrm{M}^{+}+1\right)$.

\section{$(R)$-Ethyl 2-bromo-2-fluoro-3-oxo-3-phenyl-} propanoate $(\mathbf{2 d})$

$43 \%$ yield, $66 \%$ ee. The enantiomeric excess was determined by HPLC on Regis Chiral 5 Micron with hexane/iPrOH (90:10) as the eluent. Flow rate: $1.2 \mathrm{~mL} / \mathrm{min}, \lambda=254 \mathrm{~nm} ; t_{\text {minor }}=$ $5.912 \mathrm{~min}, t_{\text {major }}=7.004 \mathrm{~min} ;{ }^{1} \mathrm{H} \mathrm{NMR}\left(\mathrm{CDCl}_{3}, 300 \mathrm{MHz}\right) \delta$ $1.28(\mathrm{t}, J=7.2 \mathrm{~Hz}, 3 \mathrm{H}), 4.38(\mathrm{q}, J=7.2 \mathrm{~Hz}, 2 \mathrm{H}), 7.48-7.53(\mathrm{~m}$, $2 \mathrm{H}), 7.64(\mathrm{~m}, 1 \mathrm{H}), 8.06-8.10(\mathrm{~m}, 2 \mathrm{H})$; APCIMS $m / z: 289.0$ $\left(\mathrm{M}^{+}+1\right)$.

\section{(S)-Ethyl 2-fluoro-3-oxo-3-phenylpropanoate (2e)}

$69 \%$ yield, $31 \%$ ee. The enantiomeric excess was determined by HPLC on (R,R)-WHELK-O1with hexane/iPrOH $(95: 5)$ as the eluent. Flow rate: $1.0 \mathrm{~mL} / \mathrm{min}, \lambda=254 \mathrm{~nm} ; t_{\text {minor }}=5.904 \mathrm{~min}$, $t_{\text {major }}=5.380 \mathrm{~min} ;{ }^{1} \mathrm{H} \mathrm{NMR}\left(\mathrm{CDCl}_{3}, 300 \mathrm{MHz}\right) \delta 1.15(\mathrm{t}, J=$ $7.2 \mathrm{~Hz}, 3 \mathrm{H}), 4.23$ (q, $J=7.2 \mathrm{~Hz}, 2 \mathrm{H}), 5.70-5.87$ (s, $J=48.9 \mathrm{~Hz}$, $1 \mathrm{H}), 7.18-7.45(\mathrm{~m}, 2 \mathrm{H}), 7.53(\mathrm{~m}, 1 \mathrm{H}), 7.94-7.98(\mathrm{~m}, 2 \mathrm{H})$; APCIMS $m / z: 211.1\left(\mathrm{M}^{+}+1\right)$.

\section{(R)-Ethyl 2-fluoro-2-cyclohexanonecarboxylate (2f)}

$73 \%$ yield, $63 \%$ ee. The enantiomeric excess was determined by HPLC on (R,R)-WHELK-O1 with hexane/iPrOH (90:10) as the eluent. Flow rate: $0.8 \mathrm{~mL} / \mathrm{min}, \lambda=210 \mathrm{~nm} ; t_{\text {minor }}$ $=10.848 \mathrm{~min}, t_{\text {major }}=12.440 \mathrm{~min} ;{ }^{1} \mathrm{H} \mathrm{NMR}\left(\mathrm{CDCl}_{3}\right.$, $300 \mathrm{MHz}) \delta 1.32(\mathrm{t}, J=7.2 \mathrm{~Hz}, 3 \mathrm{H}), 1.61-1.89(\mathrm{~m}$, $2 \mathrm{H}), 2.06-2.10(\mathrm{~m}, 1 \mathrm{H}), 2.51-2.73(\mathrm{~m}, 3 \mathrm{H}), 4.30$ (q, $J=7.2 \mathrm{~Hz}$, $2 \mathrm{H})$.

\section{(S)-Ethyl 2-(4'-methylbenzenesulfonyl)-2-fluoro-3- oxo-3-phenylpropanoate $\mathbf{( 2 g})$}

$74 \%$ yield, $78 \%$ ee. The enantiomeric excess was determined by HPLC on Venusil Chiral OD-H with hexane/iPrOH (92:8) as the eluent. Flow rate: $0.3 \mathrm{~mL} / \mathrm{min}, \lambda=254 \mathrm{~nm} ; t_{\text {minor }}=$ $27.176 \mathrm{~min}, t_{\text {major }}=24.288 \mathrm{~min} ;{ }^{1} \mathrm{H} \mathrm{NMR}\left(\mathrm{CDCl}_{3}, 300 \mathrm{MHz}\right) \delta$ $1.41(\mathrm{t}, J=7.2 \mathrm{~Hz}, 3 \mathrm{H}), 2.36(\mathrm{~s}, 3 \mathrm{H}), 4.38(\mathrm{q}, J=7.2 \mathrm{~Hz}, 2 \mathrm{H})$, 7.09-7.18 (m, 4H), 7.20-7.35 (m, 3H), 7.54 (s, 2H); APCIMS $m / z: 365.1\left(\mathrm{M}^{+}+1\right)$.

\section{(S)-Ethyl 2-(N-ethylmaleimide)-2-fluoro-3-oxo-3- phenylpropanoate $(\mathbf{2 h})$}

$78 \%$ yield, $82 \%$ ee. The enantiomeric excess was determined by HPLC on Venusil Chiral OD-H with hexane/iPrOH (94:6) as the eluent. Flow rate: $0.5 \mathrm{~mL} / \mathrm{min}, \lambda=254 \mathrm{~nm} ; t_{\text {minor }}=$ $13.832 \mathrm{~min}, t_{\text {major }}=12.432 \mathrm{~min} ;{ }^{1} \mathrm{H} \mathrm{NMR}\left(\mathrm{CDCl}_{3}, 300 \mathrm{MHz}\right) \delta$ $1.16(\mathrm{t}, J=7.2 \mathrm{~Hz}, 3 \mathrm{H}), 1.28(\mathrm{t}, J=7.2 \mathrm{~Hz}, 3 \mathrm{H}), 2.58(\mathrm{dd}, J=$ $18.3 \mathrm{~Hz}, 1 \mathrm{H}), 3.05(\mathrm{dd}, J=18.3 \mathrm{~Hz}, 1 \mathrm{H}), 3.61$ (q, $J=7.2 \mathrm{~Hz}$, $2 \mathrm{H}), 4.14(\mathrm{~m}, 1 \mathrm{H}), 4.42(\mathrm{~m}, J=7.2 \mathrm{~Hz}, 2 \mathrm{H}), 7.46-7.51$ $(\mathrm{m}, 2 \mathrm{H}), 7.62(\mathrm{~m}, 1 \mathrm{H}), 8.12(\mathrm{~m}, 2 \mathrm{H}) ;{ }^{13} \mathrm{C} \mathrm{NMR}\left(\mathrm{CDCl}_{3}\right.$, $75 \mathrm{MHz}) \delta 12.9,13.8,30.8,34.1,44.9,63.5,128.5,128.8$, $130.1,130.2,134.8,168.5,174.8$; APCIMS $m / z: 336.1$ $\left(\mathrm{M}^{+}+1\right)$. 
(S)-Ethyl 2-(N-benzylmaleimide)-2-fluoro-3-oxo-3phenylpropanoate (2i)

$83 \%$ yield, $81 \%$ ee. The enantiomeric excess was determined by HPLC on Venusil Chiral OD-H with hexane/iPrOH (92:8) as the eluent. Flow rate: $0.3 \mathrm{~mL} / \mathrm{min}, \lambda=254 \mathrm{~nm}: t_{\text {minor }}=$ $27.176 \mathrm{~min}, t_{\text {major }}=24.288 \mathrm{~min} ;{ }^{1} \mathrm{H} \mathrm{NMR}\left(\mathrm{CDCl}_{3}, 300 \mathrm{MHz}\right) \delta$ $1.27(\mathrm{t}, J=7.2 \mathrm{~Hz}, 3 \mathrm{H}), 2.58(\mathrm{dd}, J=18.3 \mathrm{~Hz}, 1 \mathrm{H}), 3.05(\mathrm{dd}, J$ $=18.3 \mathrm{~Hz}, 1 \mathrm{H}), 4.14(\mathrm{~m}, 1 \mathrm{H}), 4.42(\mathrm{~m}, J=7.2 \mathrm{~Hz}, 2 \mathrm{H}), 7.67$ (q, $J=15 \mathrm{~Hz}, 2 \mathrm{H}), 7.25-7.37$ (m, 5H), 7.46-7.51 (m, 2H), 7.62 $(\mathrm{m}, 1 \mathrm{H}), 8.12(\mathrm{~m}, 2 \mathrm{H}) ;{ }^{13} \mathrm{C} \mathrm{NMR}\left(\mathrm{CDCl}_{3}, 75 \mathrm{MHz}\right) \delta 13.8$, $30.8,42.7,45.0,45.3,63.5,128.0,128.6,128.7,128.8,130.1$, 130.2, 134.9, 174.6; APCIMS $m / z: 398.1\left(\mathrm{M}^{+}+1\right)$.

\section{Synthesis of racemic samples}

The mixture of Selectfluor $(0.057 \mathrm{~g}, 0.16 \mathrm{mmol})$ and ethyl benzoylacetate $(0.031 \mathrm{~g}, 0.16 \mathrm{mmol})$ in $\mathrm{CH}_{3} \mathrm{CN}(1 \mathrm{~mL})$ was stirred at $90{ }^{\circ} \mathrm{C}$ under microwave irradiation for $40 \mathrm{~min}$. The reaction was quenched by water. The mixture was extracted with ethyl acetate $(3 \mathrm{~mL})$. The organic layer was washed with aqueous $\mathrm{HCl}(2 \mathrm{M}, 5 \mathrm{~mL})$ and water $(5 \mathrm{~mL})$, and dried over $\mathrm{Na}_{2} \mathrm{SO}_{4}$. After evaporation of the solvent, the residue was purified by flash column chromatography ( $8: 1$ hexane/EtOAc) to give ethyl 2-methyl-2-fluoro-3-oxo-3-phenylpropanoate $(0.032 \mathrm{~g}, 94 \%)$ as a colorless oil.

\section{General procedure for recycling of C-1}

The reaction mixture was loaded onto a fluorous silica-gel cartridge $(5 \mathrm{~g})$ and eluted by $80: 20 \mathrm{MeOH} / \mathrm{H}_{2} \mathrm{O}$ to collect nonfluorous components, including the fluorinated product. The cartridge was eluted with $\mathrm{MeOH}$ to collect $\mathbf{C - 1}$. After concentration of the $\mathrm{MeOH}$ fraction and drying at $60{ }^{\circ} \mathrm{C}$ for $8 \mathrm{~h}$, the recovered promoter was ready for the next round of reactions.

\section{Supporting Information}

\section{Supporting Information File 1}

Chiral HPLC chromatograms for fluorination products 2a-i. LC-MS, NMR spectra for fluorination products $\mathbf{2 a}-\mathbf{i}$ and cinchona alkaloid derivatives C-1, C-2, C-3 and C-6. LC-MS spectra for $\mathbf{2 h}$ and HRMS spectra for $\mathbf{C}-\mathbf{1}$. [http://www.beilstein-journals.org/bjoc/content/ supplementary/1860-5397-8-138-S1.pdf]

\section{Acknowledgements}

W. Z. thanks the University of Massachusetts Boston for a grant support. W. Y. thanks for support from "NUST Excellence Initiative", NUST Research Funding (2011ZDJH07), Jiangsu Provincial Natural Science Foundation of China for Key Projects (BK2010070) and National Natural Science Foundation of China (20902047).

\section{References}

1. Banks, R. E.; Tatlow, J. C., Eds. Organofluorine Chemistry: Principles and Commercial Applications; Topics in Applied Chemistry; Plenum Press: New York, 1994.

2. Ojima, I.; McCarthy, J. R.; Welch, J. T., Eds. Biomedical Frontiers of Fluorine chemistry; ACS Symposium Series, Vol. 639; American Chemical Society: Washington, 1996. doi:10.1021/bk-1996-0639

3. Ojima, I. Fluorine in Medicinal Chemistry and Chemical Biology; Wiley-Blackwell: Hoboken, 2009.

4. Qiu, X.-L.; Xu, X.-H.; Qing, F.-L. Tetrahedron 2010, 66, 789-843. doi:10.1016/j.tet.2009.11.001

5. Phan, L. T.; Clark, R. F.; Rupp, M.; Or, Y. S.; Chu, D. T. W.; Ma, Z. Org. Lett. 2000, 2, 2951-2954. doi:10.1021/ol006226o

6. Abad, A.; Agulló, C.; Cuñat, A. C.; González-Coloma, A.; Pardo, D. Eur. J. Org. Chem. 2010, 2182-2198. doi:10.1002/ejoc.200901499

7. Banks, R. E. J. Fluorine Chem. 1998, 87, 1-17. doi:10.1016/S0022-1139(97)00127-9

8. Nyffeler, P. T.; Gonzalez Durón, S.; Burkart, M. D.; Vincent, S. P.; Wong, C.-H. Angew. Chem., Int. Ed. 2005, 44, 192-212. doi:10.1002/anie.200400648

9. Singh, R. P.; Shreeve, J. M. Acc. Chem. Res. 2004, 37, 31-44. doi:10.1021/ar030043v

10. Cahard, D.; Audouard, C.; Plaquevent, J.-C.; Roques, N. Org. Lett. 2000, 2, 3699-3701. doi:10.1021/ol006610l

11. Mohar, B.; Baudoux, J.; Plaquevent, J.-C.; Cahard, D. Angew. Chem., Int. Ed. 2001, 40, 4214-4216. doi:10.1002/1521-3773(20011119)40:22<4214::AID-ANIE4214>3.0.CO ;2-B

12. Cahard, D.; Audouard, C.; Plaquevent, J.-C.; Toupet, L.; Roques, N. Tetrahedron Lett. 2001, 42, 1867-1869. doi:10.1016/S0040-4039(01)00017-X

13. Shibata, N.; Suzuki, E.; Takeuchi, Y. J. Am. Chem. Soc. 2000, 122, 10728-10729. doi:10.1021/ja002732x

14. Shibata, N.; Suzuki, E.; Asahi, T.; Shiro, M. J. Am. Chem. Soc. 2001, 123, 7001-7009. doi:10.1021/ja010789t

15. Ma, J.-A.; Cahard, D. Chem. Rev. 2008, 108, PR1-PR43. doi:10.1021/cr800221v

16. Fukuzumi, T.; Shibata, N.; Sugiura, M.; Nakamura, S.; Toru, T. J. Fluorine Chem. 2006, 127, 548-551. doi:10.1016/j.jluchem.2006.01.004

17. Hintermann, L.; Togni, A. Angew. Chem., Int. Ed. 2000, 39, 4359-4362. doi:10.1002/1521-3773(20001201)39:23<4359::AID-ANIE4359>3.0.CO ;2-P

18. Hintermann, L.; Togni, A. Catalytic halogenation of activated methylene and methane compound. Eur. Pat. Appl. EP1151980 A1, Nov 7, 2001.

19. Muñiz, K. Angew. Chem., Int. Ed. 2001, 40, 1653-1656. doi:10.1002/1521-3773(20010504)40:9<1653::AID-ANIE16530>3.0.CO ;2-W

20. Hintermann, L.; Perseghini, M.; Togni, A. Beilstein J. Org. Chem. 2011, 7, 1421-1435. doi:10.3762/bjoc.7.166

21. Thierry, B.; Audouard, C.; Plaquevent, J.-C.; Cahard, D. Synlett 2004, 856-860. doi:10.1055/s-2004-817781

22. Baudequin, C.; Plaquevent, J.-C.; Audouard, C.; Cahard, D. Green Chem. 2002, 4, 584-586. doi:10.1039/b208817g

23. Fache, F.; Piva, O. Tetrahedron Lett. 2001, 42, 5655-5657. doi:10.1016/S0040-4039(01)01036-X

24. Kaleta, Z.; Egyed, O.; Soós, T. Org. Biomol. Chem. 2005, 3 , 2228-2230. doi:10.1039/b504973c 
25. Wang, L.; Cai, C.; Curran, D. P.; Zhang, W. Synlett 2010, 433-436. doi:10.1055/s-0029-1219198

26. Chu, Q.; Zhang, W.; Curran, D. P. Tetrahedron Lett. 2006, 47, 9287-9290. doi:10.1016/j.tetlet.2006.10.101

27. Zhang, W. Top. Curr. Chem. 2012, 308, 175-190. doi:10.1007/128_2011_257

28. Song, C. E., Ed. Cinchona Alkaloids in Synthesis and Catalysis: Ligands, Immobilization and Organocatalysis; Wiley-VCH: Weinheim, Germany, 2009.

29. Zhang, W.; Curran, D. P. Tetrahedron 2006, 62, 11837-11865. doi:10.1016/j.tet.2006.08.051

30. Fluorous SPE cartridges are available from Fluorous Technologies, Inc. http://www.fluorous.com and http://www.silicycle.com

\section{License and Terms}

This is an Open Access article under the terms of the Creative Commons Attribution License

(http://creativecommons.org/licenses/by/2.0), which permits unrestricted use, distribution, and reproduction in any medium, provided the original work is properly cited.

The license is subject to the Beilstein Journal of Organic Chemistry terms and conditions:

(http://www.beilstein-journals.org/bjoc)

The definitive version of this article is the electronic one which can be found at: doi:10.3762/bjoc. 8.138 